\title{
DYNAMIC BEHAVIOUR ANALYSIS OF EMU TRAIN ENTERING AND EXITING TUNNELS USING AERODYNAMIC LOAD DATA
}

\author{
Weixiao Wang ${ }^{1}$, Shunmei Guo ${ }^{*}$, Jinxu Liu ${ }^{2}$ \\ ${ }^{1}$ Department of Railway Vehicle, Hebei Vocational College of Rail Transportation, \\ Shijiazhuang, 052165, Hebei, China \\ ${ }^{2}$ Hebei Institute of Architectural Design \& Research Co., LTD, Shijiazhuang, 050021, Hebei, China \\ *Corresponding author: Shunmei Guo \\ Email: shunmeiabc@163.com
}

\begin{abstract}
To study the change of aerodynamic load pressure when the EMU (Electric Multiple Unit) enters and exits the tunnel, the corresponding aerodynamic equation is established. By analysing the development process of EMU trains under mechatronics, a research background for the air pressure problem caused by the speed increase of high-speed trains is displayed. Furthermore, the aerodynamic problems and related pressure waves in the tunnel of the training group are introduced. Based on the aerodynamic load data, the lateral vibration characteristics of the train are analysed. It is proposed that after the train enters the tunnel, the surrounding air generates pressure waves and expansion waves due to the restriction of the train surface and the tunnel wall. Subsequently, the Bernoulli equation is used to calculate the pressure values of the train under different running conditions in the tunnel. Finally, the ANSYS Fluent fluid calculation software is used to simulate the aerodynamics of the train tunnel of the EMU. The results show that, affected by space constraints and air compressibility, when the train enters the tunnel, the compression wave changes from three-dimensional to one-dimensional. After the head of the train enters the tunnel, the average pressure value at the cross-section of $x=2 D$ increases rapidly. At $t=0.011$ second, the average pressure of the cross-section increases slowly, and the rate of change reaches the maximum. At $\mathrm{t}=0.027$ second, the average pressure of the cross-section reaches the maximum. When the train approaches the cross-section, the pressure value at the sampling point close to the train body changes much greater than the change rate of the other two sampling points. The simulation results show that the transverse force of the train in the tunnel is $0.45 \mathrm{Kn}$. Therefore, when the train travels in the tunnel, it will be subject to more complicated aerodynamic load conditions. This study will provide references for studying the aerodynamics of high-speed trains passing through tunnels.
\end{abstract}

Keywords: Aerodynamic load; Aerodynamic effect; Electric Multiple Unit; High-speed railway tunnel; Numerical simulation.

\section{Introduction}

In recent years, China's great achievements in highspeed railway development attract worldwide attention. China has the largest high-speed railway in the world and the longest-running mileage. It is also a country with many mountains and hills. Due to the topographical conditions, the proportion of bridges and tunnels occupied is getting higher and higher in the process of constructing the railway lines [1].

Also, in response to the call of "Poverty Alleviation" and "The Belt and Road Initiative", the scale of constructing high-speed railways in the western mountainous areas is also increasing. The design and high reservation speeds need to be considered seriously as well. The number of long and extra-long tunnels is growing continuously.
In places with more complicated terrain, such as Guizhou, Xinjiang and Tibet, there will inevitably be more large and extra-long tunnel groups [2].

EMU (Electric Multiple Unit) trains are characterized by high-speed trains with large slenderness ratios running on railway lines determined on the ground, and they have the characteristics of large transportation volume, low transportation costs, high energy efficiency ratio and high punctuality [3]. With the increase of the train speed, the requirements for high-speed railway lines and tunnels and other infrastructures are also constantly improving. Because the EMU trains are exposed to sound barriers, strong crosswinds, passing cars, and tunnels during operation, the condition affects the steady-state of the train, causing severe vibration of the body and affecting the comfort and safety of passengers $[4,5]$. 
Besides, high-speed trains inevitably pass through various long tunnels in the course of traveling, which brings various complicated tunnel aerodynamic problems. The study of the pressure wave and aerodynamic load of the EMU train in the tunnel has great significance to the analysis of the traveling speed, tunnel passing capacity and stability of the EMU train.

Xiong et al. (2020) studied the aerodynamic effects caused by the tunnel when the train speed increases. And when the internal pressure fluctuation exceeds the limit, it will affect the comfort of passengers. The overall system composed of trains and tunnels is studied employing on-site measurement. The results show that tunnel entrances and exits, gas wells and train speed are important factors that cause rapid changes in the internal and external pressures of the train. When the air shaft is opened, the internal and external aerodynamic pressure changes will increase. The magnitude of the internal pressure change of the train depends on the direction of travel. Moreover, when the train passes through the tunnel interval with a gap interval of $5.5 \mathrm{~m}$ at a speed greater than $80 \mathrm{~km} / \mathrm{h}$, it will cause passengers' ear discomfort [6]. Yuan et al. (2019) analysed the aerodynamic characteristics of a quiet urban train passing through a tunnel based on sliding grid technology. The pressure curve of the two trains and the tunnel surface is obtained through numerical simulation, and the motion model is used to discuss the aerodynamic characteristics of the two trains and the tunnel surface. The pressure curve is simulated.

The experimental results show that the asymmetric internal structure of the station and the sudden change of the cross-section are the main factors that cause the poor stability of intercity trains [7]. Deng et al. (2020) explored the operational safety of high-speed trains from bridges into tunnels, and revealed the difference in the aerodynamic performance of high-temperature superconducting during the two processes in terms of transient aerodynamic load and flow field. The calculation method of dividing aerodynamic loads on the surface is proposed, and the real-life history of aerodynamic loads is obtained. The experimental results show that in the crosswind environment, the five aerodynamic coefficients of the train entering the tunnel have the largest fluctuation increase of 4.0-84.3 times. The control of the aerodynamic parameters of the rear compartment is the key to achieving traffic safety [8].

To study the aerodynamic characteristics of trains entering and leaving the tunnel, the aerodynamics and Bernoulli equation are applied to analyse and calculate the pressure conditions of trains entering and leaving the tunnel. And by decomposing the working conditions, the pressure values of the train entering the tunnel, passing through the tunnel and exiting the tunnel are calculated. Finally, the ANSYS Fluent fluid calculation software is used to simulate the CRH3 high-speed train model running at $350 \mathrm{~km} / \mathrm{h}$, and the formation and propagation characteristics of the pressure wave in the tunnel are obtained.

\section{Aerodynamic Analysis of the Following Trains under Tunnel Conditions based on Aerodynamic Load Data}

\subsection{Application of Mechatronics in the Railway Industry}

Mechatronics technology promotes the continuous development of social industrial technology and is widely used in construction machinery, becoming the highest stage of automation development [9]. Mechatronics is a technology that applies electronic technology to the main functions, power functions, information processing and control functions of mechanical structure, and combines mechanical devices with electronic design and software. This technology can be used according to the functional goals and optimization goals of the system. It can rationally display the various functional units of the system and achieve specific functions, and it is characterized by multi-function, high quality and low energy consumption $[10,11]$. Figure 1 shows the technical composition of mechatronics.

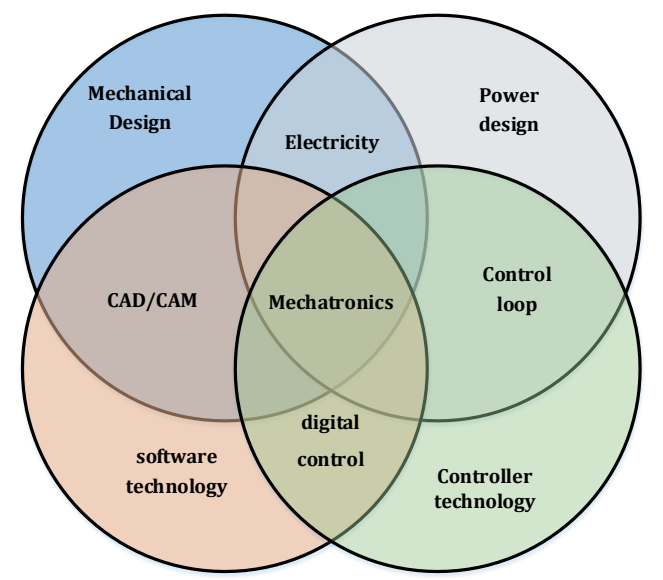

Figure 1: Mechatronics technology relationship

If the railway is the skeleton of the high-speed railway and the train is the muscle, then the electrical and automation equipment in the highspeed train is energy and nerves [12]. At the beginning of the rise of railways in the 18th century, railway trains are directly designed by mechanical designers. The railway industry develops rapidly and the track facilities are in place. However, the design and maintenance of trains restrict the development of the railway industry [13]. In this case, the mechatronics technology is used to design railway trains to reduce costs and consumption and enhance 
the railway competitiveness against other transportation industries. The technology is also used for vehicles in the wheel-rail transportation system, such as the high-speed EMUs (Electric Multiple Units), quasi-high-speed large railway vehicles, freight heavy-duty trains, trackless maglev trains, which makes mechatronics more and more widely used in the railway industry [14-17].

In the railway industry, electrified equipment can be divided into primary equipment responsible for the production, transmission, distribution and use of electrical energy according to their functions, and secondary equipment responsible for detection, measurement, control, protection and regulation, to realize the provision of energy to the railway power system [18]. The improvement of the degree of electromechanical integration in the railway industry makes the mechanical structure of EMUs more simplified, the layout more reasonable, and the vehicle performance is also improved [19]. In the modern railway industry, many complex mechanical designs are transferred to mechatronics control design, and the railway industry is gradually promoted by means of the multidisciplinary and technological intersection, accelerating the intelligent development of the railway industry [20, 21].

\subsection{Problems of Aerodynamics in Tunnels of EMUs}

The development of EMU trains brings people a more convenient life, but with the increase in the speed of EMUs, more complicated aerodynamic problems need to be tackled during the driving process [22]. Especially when EMU trains pass through at high speed in the tunnel, due to the limited airflow, the train will compress the air inside to form an initial compression wave and transmit it to the inside of the tunnel. Part of the compression wave will be refracted through the tunnel wall to the outside of the cave, forming a micro-pressure wave (Figure 2), causing the vibration of the building outside the tunnel. When the intensity is high, it will cause a sonic boom, which will affect the environment around the tunnel and the lives of residents. With the implementation of increasing the train speed, the phenomenon of micro-pressure waves at the tunnel entrance becomes more and more common, and the influence is increasingly serious $[23,24]$.

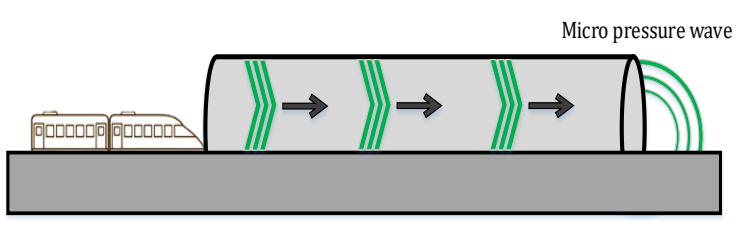

Figure 2: The low-pressure wave generated by the train of the EMU after going through the tunnel
When the EMU train enters the tunnel, the entrance wave will be generated, which will not propagate in the tunnel and is not affected by the nonlinear characteristics. However, because it is an infrasound wave, the entrance wave also causes environmental problems. The air resistance of the EMU train in traveling is the focus of aerodynamic research. With the increase of the train speed, the air resistance received continues to increase, causing the energy consumption of the train [25]. Related studies find that when high-speed trains travel at a speed of $250-300 \mathrm{~km} / \mathrm{h}$, the air resistance accounts for $75-80 \%$. The problem of air resistance becomes more prominent when passing through tunnels, and the trains are affected by aerodynamic forces, which affects the stability and safety of the running train.

When the EMU is driving into the tunnel, the airflow field around the train body changes due to the space limitation of the tunnel wall, causing the train body to swing laterally after entering the tunnel, which affects the comfort of passengers, and the mechanical performance and fatigue resistance of the train as well. When the train is running on a double track, the pressure on both sides of the train is different, which increases the energy consumption and affects the running stability of the train [26]. Therefore, when the train is running in the tunnel, the amplitude of the train body is significantly higher than that running on the open line. The micropressure wave, inlet wave and air resistance generated by the train are studied by the analysis of the aerodynamics of the train in the tunnel.

\subsection{Analysis of Train Lateral Vibration Characteristics based on Aerodynamic Load Data}

When the EMU train is running, it is closely connected with the railway track, the power supply network and the surrounding air. The interaction of these elements constitutes the EMU train dynamic system. When the train is running, the reason for the various vibrations of the EMU train is mostly the uneven railway track. And the air pressure change directly reflects the air resistance of the train body when the train is running. When the train enters and exits the tunnel, the surface air pressure of the train changes, forming the pressure wave of the tunnel. The aerodynamic effect of trains is the study of the effects of air changes on the body of the train, and it involves the application of fluid mechanics and aerodynamics in rail transit [27]. Compared with the aerodynamic system of a vehicle, due to the difference in the motion unit, the influence of the EMU train on the surrounding atmosphere is much greater than that of the car. When the train is running at high speed, the surrounding air is a complex, compressible and non-constant airflow. 
The pressure on the surface of the train changes as the speed of the train increases. In an ideal state, the pressure on the surface of the train is proportional to the square of the running speed [28].

The lateral acceleration of the EMU train is an important criterion for evaluating the smoothness of train operation and the comfort of passengers. In addition to the vibration acceleration of the centre of the train itself, it also includes the distance on the floor of the train at a distance of $10 \mathrm{~cm}$ from one side of the two endplates. The acceleration represents the lateral acceleration and vertical acceleration of the train (Figure 3).

The acceleration at points $a$ and $b$ in the figure are calculated by weighting the lateral acceleration of the centre of mass, the acceleration of the vehicle body roll, and the acceleration of the vehicle body shaking head [29]. The acceleration at this point can reflect the characteristics of the EMU train during the running process. Therefore, the data at these two points are selected as the vibration acceleration data in the test line.

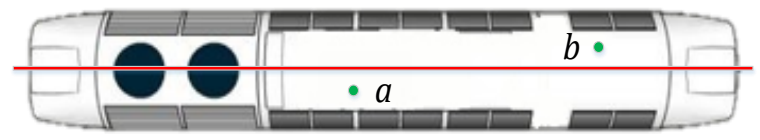

Figure 3: The lateral acceleration of the EMU train

When the EMU train is running at high speed, the air resistance increases sharply, and the aerodynamic effect produced causes huge damage to the train, resulting in a more complicated relationship between air pressure and vibration. The air pressure and vibration data of EMU trains on the railway line are taken as the research object, and the influence of the train's lateral vibration characteristics caused by the aerodynamic load of the EMU train on the stability of the train is studied. The air pressure change intuitively affects the air resistance of the body, the airlift force of the train, and the air torque. Therefore, under the conditions of the tunnel running, open-line running, and tunnel meeting, the aerodynamics loads are different.

\subsection{Calculation of the Pressure Value of EMU Trains Entering and Leaving the Tunnel under Tunnel Conditions}

Relevant studies show that the airflow caused by EMU trains entering the tunnel is three-dimensional, unsteady and compressible turbulence. After EMU trains enter the tunnel, the air of the EMU train is limited by the tunnel wall and body surface, and the accumulated air cannot pass through the train surface.
On the surface, driven by the high-speed train, the air is propelled along the direction of the train, which is called piston wind [30]. Based on the Bernoulli equation, the aerodynamic changes of EMU train entering and exiting tunnel are modelled and analysed. The equation obtained by integrating Euler's equation along a certain path in the flow field is known as the Bernoulli equation, and the equation is:

$$
z_{1}+\frac{p_{1}}{\rho g}+\frac{v_{1}^{2}}{2 g}=z_{2}+\frac{p_{2}}{\rho g}+\frac{v_{2}^{2}}{2 g}+h_{w 1 \sim 2}+\frac{1}{g} \int_{1}^{2} \frac{\partial v}{\partial t} d s
$$

In equation (1), $\rho$ represents density, $\mathrm{g}$ represents gravitational acceleration, $\mathrm{p}$ represents fluid pressure, $\mathrm{z}$ represents three-dimensional ordinate, $\mathrm{v}$ represents fluid velocity, $\mathrm{t}$ represents time, and $h_{w 1 \sim 2}$ represents resistance loss along the way.

To simplify the research model, the assumptions are as follows: the cross-sectional parameter change of the tunnel model is smaller than that of the tunnel length; The gas in the tunnel is a one-dimensional compressible fluid, and the fluid difference caused by the height is small, ignoring the gravity; the air in the tunnel has a small change in physical quantity caused by frictional heat generation, and the gas in the tunnel is a one-dimensional compressible fluid under adiabatic isentropic conditions. The Bernoulli equation for compressible fluid and the speed of the airflow is obtained:

$$
\begin{aligned}
& \frac{p_{1}}{\rho}+\frac{v_{1}^{2}}{2}=\frac{p_{2}}{\rho}+\frac{v_{2}^{2}}{2}+h_{w 1 \sim 2}+x \frac{d v}{d t} \\
& v_{s}=\frac{A_{0} v_{0}-A v}{A-A_{0}}+v_{0}=\frac{v_{0}-v}{1-\alpha}
\end{aligned}
$$

$\mathrm{V}_{0}$ represents the traveling speed of the EMU train, $\mathrm{A}_{0}$ represents the cross-sectional area of the train, $\alpha$ represents the block ratio of the train to the tunnel, A is the cross-sectional area of the tunnel, $\mathrm{v}$ is the wind speed of the piston wind, and $v_{s}$ is the speed of the air in the tunnel relative to the train.

In the tunnel, the piston wind is threedimensional and very stable, and the air-fluid parameters change as time goes by. Therefore, the piston wind pressure values in the three different states are calculated when the train enters the tunnel, and the air density in different preparation states is calculated. The density is corrected, and the equations of compression wave and expansion wave are used to analyse the condition of EMU trains after entering the tunnel. Figure 4 is a schematic diagram of different running situations under the tunnel. 
a)

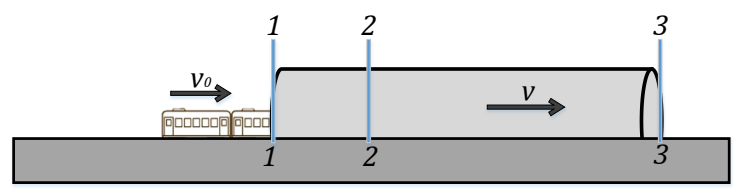

b)

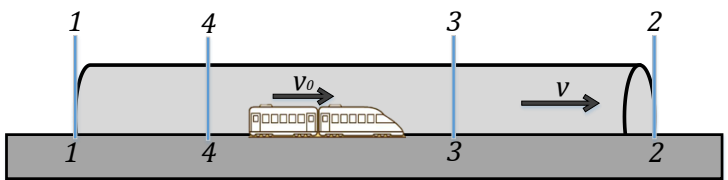

c)

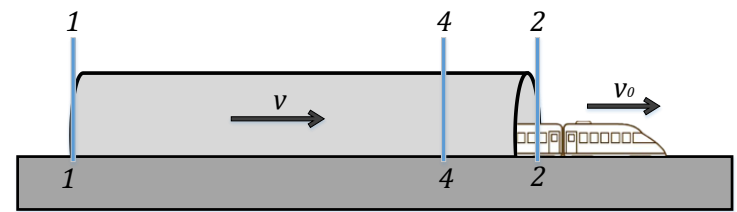

Figure 4: Process diagram of the train entering and exiting the tunnel.

(Note: a) Part of the train enters the tunnel; b) All the train enters the tunnel; c) Part of the train exits the tunnel).

(1) Part of the train enters the tunnel: when the train partly enters the tunnel (Figure 4a), the air in the tunnel is compressed on the surface, which causes a disturbance. Some of the air flows in the front of the train along the direction of the train; the other part of the air will flow out of the tunnel from the opposite direction of the train in the annular space [31]. Because the tunnel space is fixed and the air is compressible, the air in front of the train compresses waves to transmit pressure disturbances to the other end of the tunnel, and its propagation speed is close to the speed of sound. The piston wind control equation of the one-dimensional nonconstant fluid under the wind is:

$$
\begin{aligned}
& \frac{p_{2}}{\rho}=\frac{p_{a}}{\rho}+h_{13}+x \frac{d v_{s}}{d t} \\
& h_{13}=N x \frac{v_{2}^{2}}{2}
\end{aligned}
$$

$h_{13}$ represents the resistance loss at both ends of the tunnel, $\rho$ represents the air density, and $p_{a}$ represents the air pressure at the head section of the train, which is about $86 \times 10-4 / \mathrm{m}$.

Thus, the unsteady Bernoulli equation of the cross-section of the locomotive and the airflow at the exit of the tunnel concerning the tunnel is obtained:

$$
\begin{array}{r}
\frac{p_{2}}{\rho}+\frac{v^{2}}{2}=\frac{p_{a}}{\rho}+\left|\lambda \frac{L-v_{0} t}{d}+1\right| \frac{v_{2}^{2}}{2}+\left(L-v_{0} t\right) \frac{d v}{d t} \\
2\left|L+\frac{\alpha L_{0}}{(1-\alpha)}\right| \frac{d v}{d t}=K v_{0}^{2}-2 k v_{0} v+|k-| \xi+\lambda \frac{L-L_{0}}{d}+1|| v^{2}, t \in\left|\frac{L_{0}}{v_{0}}, \frac{L}{v_{0}}\right|
\end{array}
$$

$\lambda$ represents the resistance parameter along the tunnel, $d$ represents the equivalent diameter of the tunnel, $v_{0}$ represents the speed of the train, and $v$ represents the speed of airflow under pressure.

When the train partly enters the tunnel, the air in the tunnel is squeezed and disturbed, causing the uneven flow of gas and changing the density of the flowing gas. The airflow change is caused by the change of air density. The changed air density equation is:

$$
\rho^{\prime}=\frac{\rho A L-\left[v t A-(1-\alpha) \frac{\alpha v_{0}-v}{1-\alpha} A t\right] \rho}{[L-x+x(1-\alpha)] A}
$$

The compression wave transfer equation at the head section of the train and the tunnel end can be:

$$
d p=\alpha \rho d v, t \in\left|0, \frac{L_{0}}{v_{0}}\right|
$$

(2) The train travels in the tunnel (Figure 4 b): After all the train enters the tunnel totally, the speed of the train is higher than the airflow speed in the tunnel, so that the space at the end of the train cannot be filled by the air in the tunnel in time, which causes the pressure of the rear part of the train to drop suddenly and perturb the propagation surface in the form of expansion wave. And the gas in front of the train transmits pressure disturbances in the form of compression waves [32]. The equation 
However, when the train enters the tunnel, the gas in front of the train will be squeezed forward to transfer the pressure in the form of a compression wave, and the negative pressure at the rear of the train will transfer the pressure disturbance backward in the form of expansion wave, which is accompanied by the pressure wave and expansion wave, and affected by the air viscosity and tunnel wall friction in the tunnel. This causes more uneven airflow in the tunnel, and the air density in the expansion zone behind the train and the compression zone in front of the train changes. The air density equations in the compression zone and expansion zone are obtained respectively:

$$
\begin{gathered}
\rho_{R}=\frac{\rho L-\alpha \rho L_{0}-(1-\alpha) \rho v_{0} t}{L-L_{0} \alpha-v_{0} t} \\
\rho_{L}=\frac{\rho v A t-\rho \frac{\alpha v_{0}-v}{(1-\alpha)}(1-\alpha) A t}{v_{0} A t}
\end{gathered}
$$

The revised pressure equation before and after the train:

$$
\begin{aligned}
& \frac{p_{3}}{\rho_{R}}+\frac{v^{2}}{2}=\frac{p_{a}}{\rho_{R}}+\left|1+\lambda \frac{L_{32}}{d}\right| \frac{v^{2}}{2}+L_{32} \frac{d v}{d t} \\
& \frac{p_{3}}{\rho_{L}}+\frac{v^{2}}{2}=\frac{p_{4}}{\rho_{R}}+\left|\xi+\lambda \frac{L_{14}}{d}\right| \frac{v^{2}}{2}+L_{14} \frac{d v}{d t}
\end{aligned}
$$

$\xi$ represents the coefficient.
In the compression zone, the pressure wave is transmitted in a form similar to a single wave, while in the expansion zone, the direction of expansion wave transmission is bidirectional, and affects the compression zone on both sides of the train. This doesn't last long and can be ignored. Finally, the transfer equations of compression wave and expansion wave are achieved:

$$
\begin{aligned}
& d p=\alpha \rho_{R} d v \\
& d p=-\alpha \rho_{L} d v
\end{aligned}
$$

(3) The train partially exits the tunnel: when the train exits the tunnel (Figure 4c), the pressure disturbance will increase briefly, and then be consumed by the roadbeds in the tunnel. And the air disturbance gradually weakens till disappears, which can be deduced the equation:

After the train leaves the tunnel, due to the influence of air viscosity and the friction of the tunnel wall, the air in the tunnel continues to move with the movement of the train for some time. Therefore, the density equation in the tunnel at this time is corrected as:

$$
\rho_{L}=\frac{\rho v A t-\rho \frac{\alpha v_{0}-v}{(1-\alpha)}(1-\alpha) A t}{v_{0} A t}
$$

The pressure equation at the rear of the train is obtained:

$$
\frac{p_{a}}{\rho_{L}}=\frac{v^{2}}{2}+\frac{p_{4}}{\rho_{L}}+\left|\xi+\lambda \frac{L-\left(L+L_{0}-v_{0} t\right)}{d}\right| \frac{v^{2}}{2}+\left(L+L_{0}-v_{0} t\right) \frac{d v}{d t}
$$

The single-wave transmission equation from the rear of the train to the expansion zone at the entrance of the tunnel is obtained:

$$
d p=-\alpha \rho_{L} d v
$$

The aerodynamic force of the train is relatively stable when the train is traveling on the open line, and the traveling speed has little effect on the vibration stability of the train.

When the train is entering and exiting the tunnel, the air pressure of the train causes severe fluctuations, and the aerodynamic pressure generated by the train cannot be ignored. In the light of the side force of the train, the body vibration of the train under this working condition is largely caused by the fluctuation of air pressure.

\subsection{Numerical Simulation of the Aerodynamics of Train Tunnels in EMUs}

The ANSYS Fluent fluid calculation software is used to do the experiment. The software can solve Euler equations and provide a variety of turbulence calculation models, which can completely simulate viscous fluids.

When the boundary between the EMU train and the tunnel wall ARE PROCESSED, the moving surface method is adopted, that is, another moving surface is set in the variable cross-section of the train and the tunnel. Real-time storage and transient analysis are conducted when the solution is being worked out, using not only two-dimensional analysis but also three-dimensional analysis. The software also provides separate and coupled solvers. 
The separation solver can solve the equations one by one in order, while the coupled can solve multiple continuous equations, momentum equations and energy equations simultaneously, and calibrate and solve scalar quantities such as turbulence one by one. The CRH3 high-speed train model running at $350 \mathrm{~km} / \mathrm{h}$ is selected as the research object to study the formation and spatial characteristics of the initial compression wave.

\section{Analysis of the Aerodynamic Load Pressure of the Following Vehicles under Tunnel Conditions \\ 3.1 Distribution Characteristics of Compression Waves in Tunnels}

A series of measurement points are placed in the tunnel along the tunnel direction.
The location of the test points is shown in the figure. The height of point A in the tunnel is $0.15 \mathrm{D}$, and the discovery distance from the tunnel wall is 0.0047D. Point $B$ is located at the top of the centreline of the tunnel.

The picture shows the pressure distribution curve of each measurement point along the length of the tunnel at different times. a $\sim \mathrm{g}$ indicate different periods. At time a, the distance between the train and the entrance of the tunnel is a nose length; and at time $b$, the train head is arriving at the tunnel entrance. $c \sim g$ is the distance between the front of the train and the tunnel entrance, and the distance is $1,3,5,7$, and 9 nose lengths respectively. Figure 5 shows the formation and the transmission characteristics of compression waves in space.

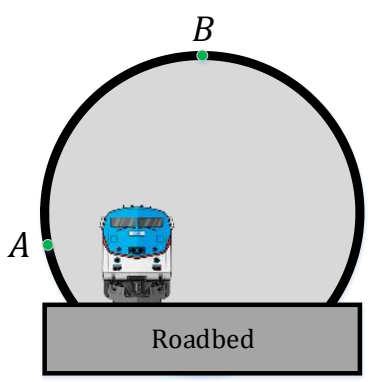

a)

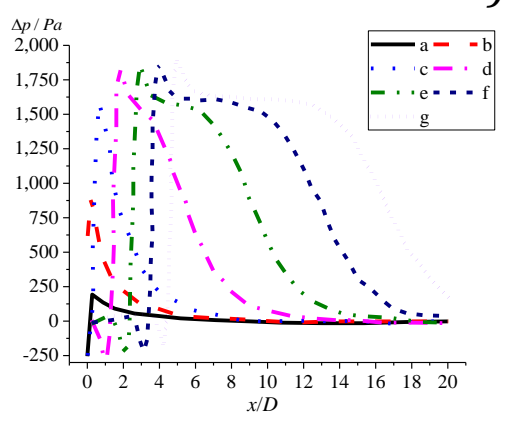

b)

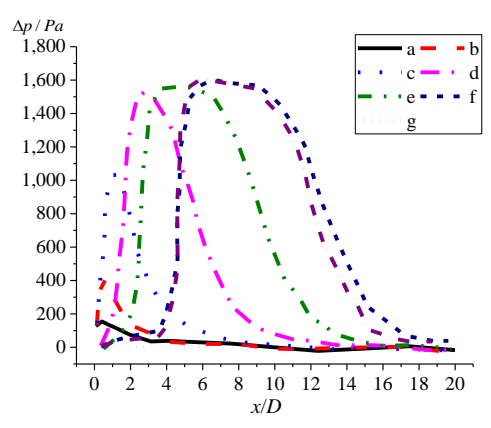

c)

Figure 5: Formation and transmission characteristics of compression waves in space. (a) tunnel sampling point setting; b) point A pressure value; c) point B pressure value)

Curve a shows that when the head of the train enters the tunnel, the air pressure in the tunnel gradually increases; while in curves $b \sim d$, the pressure in the front of the train increases continuously after entering the tunnel, and the pressure at the front of the train is more intense, which bears the three-dimensional characteristics. In curves $\mathrm{e} \sim \mathrm{g}$, the train is about 5 nose lengths away from the tunnel entrance, and the air pressure distribution around the front and the body remains unchanged, showing one-dimensional characteristics.
Due to the compressibility of the air and the space constraints in the train and tunnel, a threedimensional initial compression wave is formed along the tunnel. When the train runs more than 5 nose lengths, the compression wave changes from a three-dimensional wave to a one-dimensional wave.

To display the distribution characteristics of the initial compression wave clearly, the average pressure and change rate at 4 nose-length sampling points from the entrance of the tunnel are analysed, and the results are shown in Figure 6. 


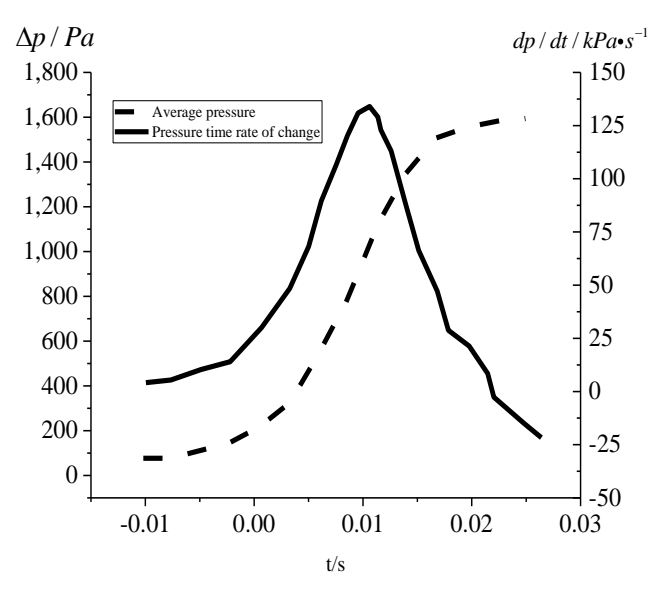

Figure 6: Average pressure and the pressure-time change rate of the $x=2 D$ section in the tunnel
Figure 6 reflects that after the train entered the tunnel, the air in the tunnel began to be disturbed, and the pressure value at the sampling point begins to increase slowly. When the train begins to enter the tunnel, the pressure in the tunnel begins to increase at a faster rate, forming the initial compression wave at the tunnel entrance. And the wave is transmitted in the tunnel. After the head of the train enters the tunnel, the average pressure at the cross-section of $\mathrm{x}=2 \mathrm{D}$ increases rapidly. At $t=0.011$ second, the increase in the average pressure of the cross-section slows down and the rate of change reaches the maximum. At $t=0.027$ second, the average pressure of the cross-section reaches the maximum.

Figures 7-9 show the comparison results of the initial compression waves at different sampling points at the $\mathrm{x}=2 \mathrm{D}$ cross-section.

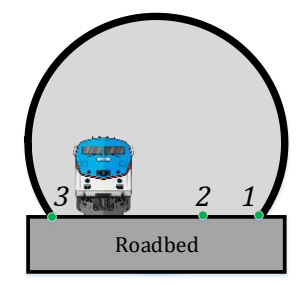

a)

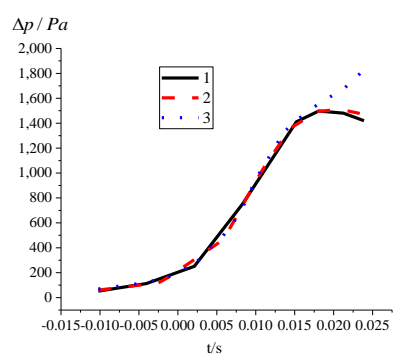

b)

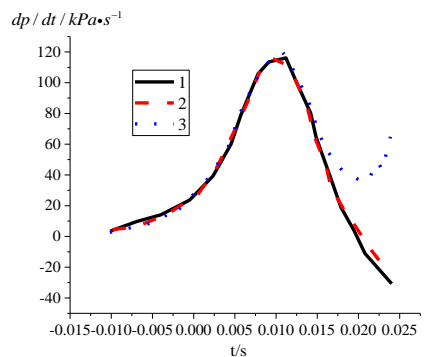

c)

Figure 7: Pressure and time rate of pressure change at the sampling point on the ground of the tunnel

a) location of the sampling point of the tunnel; b) pressure value of the sampling point; c) time rate of the pressure changes at the sampling point)

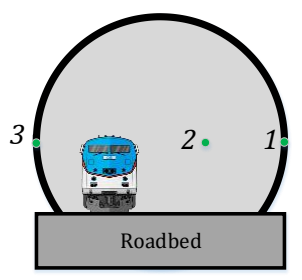

a)

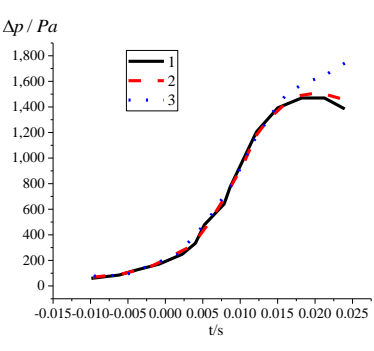

b)

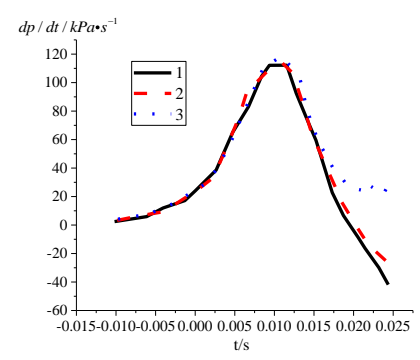

c)

Figure 8: Pressure and time rate of pressure change at the sampling point in the middle of the tunnel. 


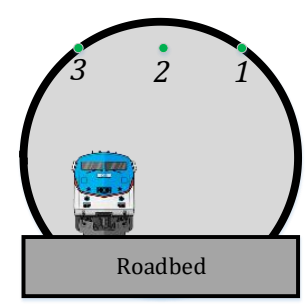

a)

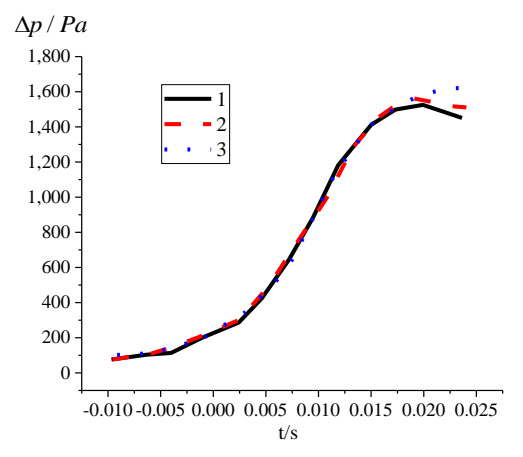

b)

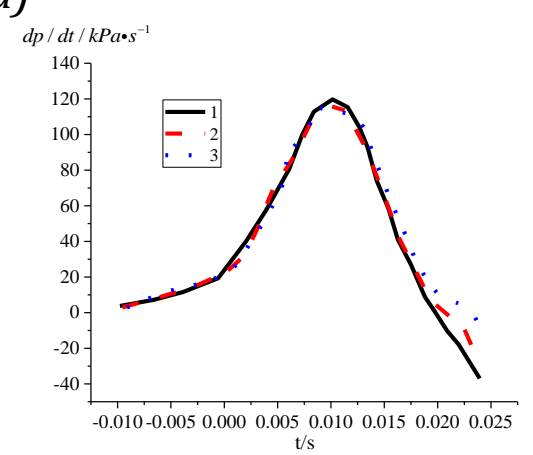

c)

Figure 9: Pressure and time rate of pressure change at the sampling point at the top of the tunnel.

Figure 7 indicates that the three sampling points are all arranged on the tunnel ground. When the train enters the tunnel, the data of the three sampling points are increasing, and the corresponding time rate of the pressure change first increases and then decreases. When the train approaches the cross-section, the pressure change value at the sampling point close to the train body is much greater than the change rate at the other two sampling points. And the corresponding decrease in the time rate of pressure change is also less than that of the other two sampling points.
In Figure 8 and Figure 9, the pressure value changes collected at the sampling points at different locations of the tunnel are similar to the trend in Figure 7. The difference is manifested as the pressure value change caused by the height change.

\subsection{Simulation of the Aerodynamic Load of EMU Train Passing Through the Tunnel}

The ANSYS Fluent fluid dynamics simulation software is used to analyse the lateral force of the train body passing through the tunnel, as shown in Figure 10.

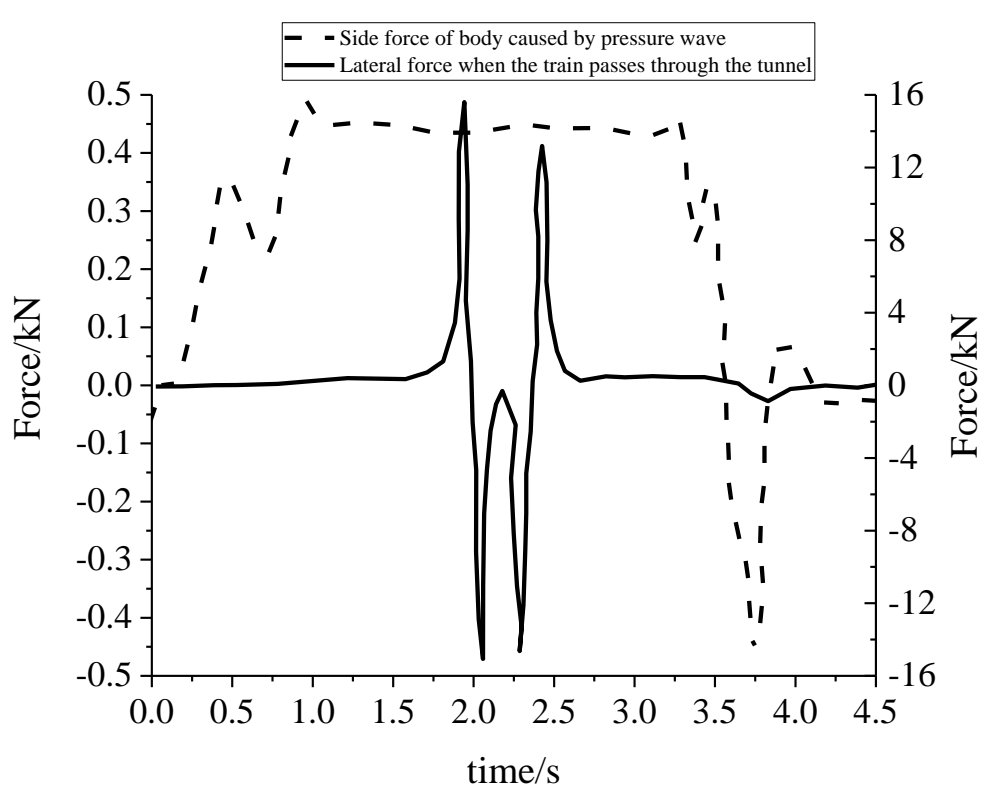

Figure 10: The lateral force of the tunnel pressure wave on the train body. 
Figure 10 shows that when the train enters the tunnel, the lateral force and roll torque received by the train body increase rapidly. After the train enters the tunnel totally, the lateral force and roll torque remain unchanged. After the train is exiting the tunnel, the lateral force and rolling torque drop sharply, and then return to the state before entering the tunnel. The curve tells that the lateral force of the train in the tunnel is $0.45 \mathrm{kN}$, which is about $0.4 \sim 0.5 \mathrm{kN}$ larger than that of the open line. The pressure on the train body is getting multiple, and the range of change will be greater. When the train meets another train in the tunnel, the protruding end boundary of the front and rear sections of the train will increase, causing the pressure in the tunnel to rise sharply and generating pressure waves and expansion waves, which transmit repeatedly in the tunnel.

\section{Conclusion}

Aerodynamics and Bernoulli equation are used to analyse the characteristics of generating pressure waves when the trains of the EMU (Electric Multiple Unit) under tunnel conditions are passing through and exiting the tunnel. Based on the aerodynamic load data, the lateral acceleration of the train is analysed, and the factors that affect the stability of the train are obtained. The experimental results show that when the train enters the tunnel, the surrounding air density will change, generating a pressure wave, and transmitting along the tunnel, and affecting the form stability of the train. Generally speaking, when two tunnels are same in height, the closer the distance to the train is, the more obvious the pressure change of the initial compression wave is. And the greater the pressure value closer to the ground is, the smaller the pressure change value near the top of the tunnel is. The pressure measurement in the middle of the tunnel is between that of the ground and the top. The pressure change rate near the train sampling point starts to increase significantly after 0.02 seconds. As the train travels in the tunnel, it is greatly affected by the positive pressure from the train head. Therefore, when the train passes through the tunnel, the pressure wave generated will affect the stability of the train due to the limitation of the train surface and the tunnel wall.

However, there are still some shortcomings. The tunnel conditions studied do not include train meetings in the tunnel. This faces a more complex air environment and has a greater impact on trains. Therefore, it will be studied in subsequent studies.

\section{References}

[1]Hu X., Deng Z., Zhang W. (2021) Effect of cross passage on aerodynamic characteristics of superhigh-speed evacuated tube transportation. Journal of Wind Engineering and Industrial Aerodynamics, 211, 104562.

[2] Iliadis P., Soper D., Baker C, et al. (2019) Experimental investigation of the aerodynamics of a freight train passing through a tunnel using a moving model. Proceedings of the Institution of Mechanical Engineers, Part F: Journal of Rail and Rapid Transit, 233(8), 857-868.

[3] Wang J., Wang T., Yang M, et al. (2021) Effect of localized high temperature on the aerodynamic performance of a high-speed train passing through a tunnel. Journal of Wind Engineering and Industrial Aerodynamics, 208, 104444.

[4] Jiang Z., Liu T., Chen X, et al. (2019) Numerical prediction of the slipstream caused by the trains with different marshalling forms entering a tunnel. Journal of Wind Engineering and Industrial Aerodynamics, 189, 276-288.

[5] Meng S., Zhou D., Wang Z. (2019) Moving model analysis on the transient pressure and slipstream caused by a metro train passing through a tunnel. Plos one, 14(9), e0222151.

[6] Xiong X., Zhu L., Zhang J, et al. (2020) Field measurements of the interior and exterior aerodynamic pressure induced by a metro train passing through a tunnel. Sustainable Cities and Society, 53, 101928.

[7] Yuan H., Zhou D., Meng S. (2019) Study of the unsteady aerodynamic performance of an intercity train passing through a station in a tunnel. Tunnelling and Underground Space Technology, 86, 1-9.

[8] Deng E., Yang W., He X, et al. (2020) Transient aerodynamic performance of high-speed trains when passing through an infrastructure consisting of tunnel-bridge-tunnel under crosswind. Tunnelling and Underground Space Technology, 102, 103440.

[9] Huang S., Li Z., Yang M. (2019) Aerodynamics of high-speed maglev trains passing each other in open air. Journal of Wind Engineering and Industrial Aerodynamics, 188, 151-160.

[10] Yang W., Deng E., Lei M, et al. (2019) Transient aerodynamic performance of high-speed trains when passing through two windproof facilities under crosswinds: A comparative study. Engineering Structures, 188, 729-744.

[11] Zhang J., Zhang M., Li Y, et al. (2019) Aerodynamic effects of subgrade-tunnel transition on high-speed railway by wind tunnel tests. Wind and Structures, 28(4), 203-213.

[12] Liang X F., Li X B., Chen G, et al. (2020) On the aerodynamic loads when a high-speed train passes under an overhead bridge. Journal of Wind 
Engineering and Industrial Aerodynamics, 202, 104208.

[13] Deng E., Yang W., He X, et al. (2021) Aerodynamic response of high-speed trains under crosswind in a bridge-tunnel section with or without a wind barrier. Journal of Wind Engineering and Industrial Aerodynamics, 210, 104502.

[14] Luo J., Li Z., Wang L, et al. (2020) Aerodynamic effect of cross passages at the entrance section of a high-speed railway tunnel in a region with mountains and canyons. Journal of Wind Engineering and Industrial Aerodynamics, 204, 104268.

[15] Deng E., Yang W., Deng L, et al. (2020) Timeresolved aerodynamic loads on high-speed trains during running on a tunnel-bridge-tunnel infrastructure under crosswind. Engineering Applications of Computational Fluid Mechanics, 14(1), 202-221.

[16] Liu T., Jiang Z., Li W, et al. (2019) Differences in aerodynamic effects when trains with different marshalling forms and lengths enter a tunnel. Tunnelling and Underground Space Technology, 84, 70-81.

[17] Li W., Liu T., Huo X, et al. (2019) Influence of the enlarged portal length on pressure waves in railway tunnels with cross-section expansion. Journal of Wind Engineering and Industrial Aerodynamics, 190, 10-22.

[18] Liu X., Zhang J., Thompson D, et al. (2021) Aerodynamic noise of high-speed train pantographs: Comparisons between field measurements and an updated component-based prediction model. Applied Acoustics, 175, 107791.

[19] Niu J., Sui Y., Yu Q, et al. (2019) Numerical study on the impact of Mach number on the coupling effect of aerodynamic heating and aerodynamic pressure caused by a tube train. Journal of Wind Engineering and Industrial Aerodynamics, 190, 100-111.

[20] Liu T., Jiang Z., Chen X, et al. (2019) Wave effects in a realistic tunnel induced by the passage of high-speed trains. Tunnelling and Underground Space Technology, 86, 224-235.

[21] Du J., Fang Q., Wang G, et al. (2021) Fatigue damage and residual life of secondary lining of high-speed railway tunnel under aerodynamic pressure wave. Tunnelling and Underground Space Technology, 111, 103851.

[22] Dong T., Minelli G., Wang J, et al. (2020) The effect of ground clearance on the aerodynamics of a generic high-speed train. Journal of Fluids and Structures, 95, 102990.
[23] Li W., Liu T., Chen Z, et al. (2020) Comparative study on the unsteady slipstream induced by a single train and two trains passing each other in a tunnel. Journal of Wind Engineering and Industrial Aerodynamics, 198, 104095.

[24] Kwon H., Jin Y., Lee W., et al. (2020) The Feasibility of Adapting Air Compressor to a HighSpeed Train to Attenuate the Aerodynamic Problems in Tunnel. International Journal of Aeronautical and Space Sciences, 21(3), 638-646.

[25] Deng E., Yang W., Lei M, et al. (2019) Aerodynamic loads and traffic safety of highspeed trains when passing through two windproof facilities under crosswind: A comparative study. Engineering Structures, 188, 320-339.

[26] Liu F., Zhou W., Niu J, et al. (2019) Impact of increased linings on pressure transients induced by a train passing through a tunnel. Sustainable cities and society, 45, 314-323.

[27] Li Y., Zhang J., Zhang M, et al. (2019) Aerodynamic effects of viaduct-cutting connection section on high-speed railway by wind tunnel tests. Journal of Aerospace Engineering, 32(5), 05019002.

[28] Zhang L., Yang M., Niu J, et al. (2019) Moving model tests on transient pressure and micropressure wave distribution induced by train passing through tunnel. Journal of Wind Engineering and Industrial Aerodynamics, 191, 121.

[29] Chen Z., Liu T., Li W, et al. (2021) Aerodynamic performance and dynamic behaviors of a train passing through an elongated hillock region beside a windbreak under crosswinds and corresponding flow mitigation measures. Journal of Wind Engineering and Industrial Aerodynamics, 208, 104434.

[30] Muñoz-Paniagua J., García J. (2019) Aerodynamic surrogate-based optimization of the nose shape of a high-speed train for crosswind and passing-by scenarios. Journal of Wind Engineering and Industrial Aerodynamics, 184, 139-152.

[31] Yang W., Deng E., Zhu Z, et al. (2020) Deterioration of dynamic response during highspeed train travelling in tunnel-bridge-tunnel scenario under crosswinds. Tunnelling and Underground Space Technology, 106, 103627.

[32] Meng S., Li X., Chen G, et al. (2021) Numerical simulation of slipstreams and wake flows of trains with different nose lengths passing through a tunnel. Tunnelling and Underground Space Technology, 108, 103701. 